\section{Antibiotic treatment and coronary heart disease}

Having established a link between Chlamydia pneumoniae infection and atherosclerosis, investigators have been prompted to ask whether antibiotic treatment might be beneficial in patients with coronary heart disease. Results of a recent US trial have shown, however, that treatment with azithromycin had no clinically significant effect on the risk of secondary cardiac events.

The Azithromycin and Coronary Events Study (ACES) included 4,012 patients with stable coronary heart disease. Participants were randomized in a 1:1 ratio to receive a weekly dose of azithromycin $(600 \mathrm{mg})$ or placebo for 1 year.

During the mean follow-up period of 3.9 years, the primary endpoint (death due to coronary heart disease, coronary revascularization, nonfatal MI, or hospitalization for unstable angina) occurred in a similar proportion of patients in the treatment and placebo groups. The frequencies of each of the components of this endpoint were also similar in the two groups.

The authors comment that the trial was designed to study the effect of azithromycin treatment in patients with late-stage coronary heart disease only, and so the results do not exclude the possibility that $C$. pneumoniae is involved in the early development or progression of atherosclerosis. They conclude, however, that "antibiotic treatment cannot be recommended for the treatment of chronic coronary heart disease".

Original article Grayston JT et al. (2005) Azithromycin for the secondary prevention of coronary events. N Engl J Med 352: $1637-1645$

\section{Magnesium can prevent postoperative atrial fibrillation}

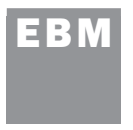

Several clinical trials have investigated the role of magnesium in preventing atrial fibrillation following cardiac surgery, but results have been mixed. Miller and colleagues have now performed a meta-analysis of these studies; their findings indicate that magnesium is indeed an effective prophylactic treatment in this setting.
The team performed a literature search to identify all relevant studies published between 1966 and July 2003. Twenty randomized trials were finally included in the analysis. These ranged in size from 20 to 400 patients and included 2,490 patients altogether. In each case, magnesium was administered preoperatively, intraoperatively or postoperatively to patients undergoing valve surgery or coronary artery bypass grafting.

The pooled data showed that postoperative atrial fibrillation occurred in $28 \%$ of patients in the placebo group, compared with only $18 \%$ of those receiving magnesium (odds ratio $0.54,95 \% \mathrm{Cl} 0.38-0.75)$. Further analysis revealed that a low dose of magnesium $(<35 \mathrm{mmol})$ was more effective than higher doses for the prevention of atrial fibrillation, although some individual studies indicated that the reverse was true. With respect to the timing of magnesium therapy, preoperative administration was most effective; this might have been because hypomagnesemia sometimes occurs before surgery. Finally, in-hospital mortality and the length of hospital stay did not appear to be affected by magnesium administration.

Original article Miller S et al. (2005) Effects of magnesium on atrial fibrillation after cardiac surgery: a meta-analysis. Heart 91: 618-623

\section{Heritability of hypertension and stroke}

Flossmann and Rothwell, from the Stroke Prevention Research Unit at the Radcliffe Infirmary in Oxford, UK, have recently published their report on family history of stroke in patients with transient ischemic attack. Their findings suggest that familial susceptibility to cerebral ischemia is linked to familial predisposition to hypertension.

The work was based on two population-based stroke incidence studies (the Oxfordshire Community Stroke Project and the Oxford Vascular Study) and two prospective, hospital-referred transient ischemic attack patient series (collected from 1976 to 1986 and 2002 to 2003). In each cohort, family history of stroke and myocardial infarction was collected from patients and their relatives using a questionnaire, and medical records were 